\title{
Conditional Probability and A Posteriori States in Quantum Mechanics*)
}

\author{
By \\ Masanao Ozawa
}

\begin{abstract}
In order to develop a statistical theory of quantum measurements including continuous observables, a concept of a posteriori states is introduced, which generalizes the notion of regular conditional probability distributions in classical probability theory. Its statistical interpretation in measuring processes is discussed and its existence is proved. As an application, we also give a complete proof of the Davies-Lewis conjecture that there are no (weakly) repeatable instruments for non-discrete observables in the standard formulation of quantum mechanics, using the notion of a posteriori states.
\end{abstract}

\section{§1. Introduction}

In the conventional theory of ideal measurements of discrete observables of quantum systems, the state of an observed system at the instant after the measurement leads a definite value is determined by the repeatability hypothesis and the resulting state change is called the reduction of wave packets. However, the repeatability hypothesis has been known to be doubtful, if we require that the processes of measurements are described in quantum mechanics. In fact, Wigner [20] pointed out that if there is an additive conservation law throughout the measuring process then the measurement of the quantity which does not commute with the conserved quantity cannot satisfy the repeatability hypothesis; see Araki-Yanase [1], Ozawa [13] for general proofs of the above statement. Moreover, we have shown in [13] that measurements of continuous observables cannot statisfy the repeatability hypothesis if the measuring process can bc

*) The results in Sections 3-4 were previously announced in part at IV USSR-Japan Symposium on Probability Theory and Mathematical Statistics, Tbilisi, USSR, 1982.

* Communicated by H. Araki, October 12, 1983. Revised June 7, 1984.

Department of Information Sciences, Tokyo Institute of Technology, Oh-okayama, Meguro-ku, Tokyo, Japan. 
described in quantum mechanics.

These results seem to show that if we shall investigate a quantum measurement not as only an ideal matter but as a quantum mechanical intervention consistent with the Hamiltonian formalism, we should abandon the repeatability hypothesis, as proposed by Davies-Lewis [7]. Such an approach to quantum measurements is much statistical in nature, and yet mathematical tools for those investigations have not been provided enough so far.

The purpose of this paper is to provide a concept of conditional probability naturally arising in measurements of continuous quantum observables. A concept of conditional expectation in quantum measurements appeared first in Nakamura-Umegaki [11], and formal theory of such conditional expectation has been much developed by Umegaki [19] in the theory of operator algebras. However, this concept of conditional expectation can be applied only to measurements of discrete observables as shown by Arveson [2]. Thus we must begin with the original statistical interpretation of quantum mechanical conditional probability in quantum measuring processes. Our approach of determining the a posteriori states from an a priori state is a variant of Bayes principle in probability theory which first appeared in von Neumann [12; pp. 337-346] in quantum mechanics.

In Section 2, we review the mathematical description of quantum mechanical measuring processes obtained in our previous paper [13]. In Section 3, the statistical interpretation of conditional probability in quantum measuring processes is investigated and the concept of a posteriori states is established in quamtum mechanics as well as their existence. In Section 4, the concept of a posteriori states is considered in a more general framework of operational quantum probability theory and some conditions for their existence are given. In Section 5, the close relation between the existence of a posteriori states and the non-existence of repeatable measurements for continuous observables is established. In particular, we shall give a complete proof of the Davies-Lewis conjecture [7; p. 247] that in the standard formulation of quantum mechanics there are no (weakly) repeatable instruments for non-discrete observables.

In Davies [6; pp. 57-60], an attempt to resolve the conjecture is done by somewhat indirect manner through the conventional concept of quantum mechanical conditional expectation. But, the new concept of conditional probability, as will be developed in this paper, seems indispensable for the complete solution. 
Our concept of a posteriori states has close connection with Cycon and Hellwig's conditional expectation [5] in operational quantum probability theory. We shall clear this connection in the forthcoming paper. Applications of the notion of a posteriori states to determining the amount of information obtained from quantum measurements also will be discussed elsewhere.

\section{$\S 2$. State Changes Caused by Measuring Processes}

In [13], the following description of a measuring process of an observable with continuous spectrum of a quantum system is considered. The observed system $I$ and the apparatus $I I$ are described by separable Hilbert spaces $\mathscr{H}_{I}$ and $\mathscr{H}_{I I}$ respectively. Let $X$ be an observable in $I$ to be measured and let $\tilde{X}$ be the observable in $I I$ to show the value of $X$ on a scale, i.e., the position of the pointer of the measuring apparatus. Denote by $X(d x)$ and $\tilde{X}(d x)$ their spectral measures respectively. The measurcment is carried out by the interaction during a finite time interval from time 0 to $t$. Let $H_{I}$ and $H_{I I}$ be the Hamiltonians of the systems $I$ and $I I$ respectively and let $H_{i n t}$ be the interaction between $I$ and $I I$. Then the time evolution of the composite system $I+I I$ from time 0 to $t$ is described by the unitary operator $U$ on $\mathscr{H}_{I} \otimes \mathscr{H}_{I I}$ such that

$$
U=\exp \left(-i t\left(H_{I} \otimes 1+1 \otimes H_{I I}+H_{i n t}\right)\right) .
$$

At the instant before the interaction the observed system $I$ is supposed to be in the (unknown) state $\rho$ and the apparatus $I I$ to be in the (prepared) state $\sigma$, where $\rho$ and $\sigma$ are density operators on $\mathscr{H}_{I}$ and $\mathscr{H}_{I I}$ respectively. Then the composite system $I+I I$ is in $\rho \otimes \sigma$ at time 0 and in $U(\rho \otimes \sigma) U^{*}$ at time $t$ by the interaction. The result of this measurement can be predicted by the probability distribution $\operatorname{Prob}(\tilde{X} \in d x)$ of the value of observable $\tilde{X}$ at time $t$ such that

$$
\operatorname{Prob}(\tilde{X} \in d x)=\operatorname{Tr}\left[U(\rho \otimes \sigma) U^{*}(1 \otimes \tilde{X}(d x))\right] .
$$

We may interprete this result to be the value of the measured observable $X$ at the initial state $\rho$. Thus we should impose the requirement

$$
\operatorname{Tr}\left[U(\rho \otimes \sigma) U^{*}(1 \otimes \widetilde{Y}(d x))\right]=\operatorname{Tr}[\rho X(d x)] .
$$

Eq. (2.3) is our sole requirement for the above interaction to be a measurement of $X$ in the initial state $\rho$. We shall call any 4-tuple $\left\langle\mathscr{H}_{I I}, \tilde{X}, \sigma, U\right\rangle$ consisting of a separable Hilbert space $\mathscr{H}_{I I}$, a self-adjoint operator $\tilde{X}$ on $\mathscr{H}_{I I}$, a density operator $\sigma$ on $\mathscr{H}_{I I}$ and a unitary operator $U$ on $\mathscr{H}_{I} \otimes \mathscr{H}_{I I}$ satisfying Eq. (2.3) for any 
density operator $\rho$ on $\mathscr{H}_{I}$ to be (a mathematical description of) a measuring process of an observable $X$ in the observed system $I$ (cf. [13; Definition 3.1]).

By the above description of a measuring process, we can determine the state change of the system $I$ caused by this measurement. Let $B$ be a Borel set of the real line. Denote by $\rho_{B}$ the state, at the instant after this measurement, of the subensemble of the measured system $I$ in which the result of this measurement lies in $B$. The state change of the system $I$ caused by this measurement is the transformation $\rho \rightarrow \rho_{B}$ for any density operator $\rho$ in $I$ and any Borel set $B$ such that $\operatorname{Tr}[\rho X(B)] \neq 0$. In particular, the usual state change $\rho \rightarrow \rho^{\prime}$ of the whole ensemble is determined by $\rho^{\prime}=\rho_{\boldsymbol{R}}$; we call the transformation $\rho \rightarrow \rho_{\boldsymbol{R}}$ the total state change of the system $I$ caused by this measurement. In order to determine the state $\rho_{B}$, suppose that the observer were to measure the simultaneously measurable observables $Y$ in $I$ and $\tilde{X}$ in $I I$ at time $t$, where $Y$ is an arbitrary observable in $I$ (cf. [12; p. 440]). Then we have the joint probability distribution of the values of $Y$ and $\tilde{X}$ :

$$
\operatorname{Prob}(Y \in d y, \tilde{X} \in B)=\operatorname{Tr}\left[U(\rho \otimes \sigma) U^{*}(Y(d y) \otimes \tilde{X}(B))\right],
$$

and the conditional probability distribution of the value of $Y$ conditioned by the probabilistic event $\tilde{X} \in B$.

$$
\operatorname{Prob}(Y \in d y \mid \tilde{X} \in B)=(1 / \operatorname{Tr}[\rho X(B)]) \operatorname{Tr}\left[U(\rho \otimes \sigma) U^{*}(Y(d y) \otimes \tilde{X}(B))\right],
$$

for $B$ such that $\operatorname{Prob}(\tilde{X} \in B) \neq 0$. Eq. (2.5) is calculated by the following formula in the usual probability theory:

$$
\operatorname{Prob}(Y \in d y \mid \tilde{X} \in B)=\operatorname{Prob}(Y \in d y, \tilde{X} \in B) / \operatorname{Prob}(\tilde{X} \in B) .
$$

On the other hand, we should adopt the following statistical interpretation of the state $\rho_{B}$ :

$$
\operatorname{Prob}(Y \in d y \mid \tilde{X} \in B)=\operatorname{Tr}\left[\rho_{B} Y(d y)\right],
$$

since the right hand side can be interpreted as the probability distribution of the observable $Y$ at the state $\rho_{B}$ and since the condition $\tilde{X} \in B$ implies the considered ensemble is in the state $\rho_{B}$. By the arbitrariness of $Y$, we can determine the state $\rho_{B}$ uniquely by Eq. (2.5) and Eq. (2.7) as follows:

$$
\rho_{B}=(1 / \operatorname{Tr}[\rho X(B)]) \operatorname{Tr}^{I I}\left[U(\rho \otimes \sigma) U^{*}(1 \otimes \widetilde{X}(B))\right],
$$

where $\operatorname{Tr}^{I I}$ is the partial trace over $\mathscr{H}_{I I}$ (cf. [13; Lemma 2.1, Eq. (3.9)]). Therefore, we have determined the state change $\rho \rightarrow \rho_{B}$ by Eq. (2.8). The total state 
change $\rho \rightarrow \rho^{\prime}=\rho_{\boldsymbol{R}}$ is obtained by putting $B=\mathbb{R}$ as follows:

$$
\rho^{\prime}=\rho_{R}=\operatorname{Tr}^{I I}\left[U(\rho \otimes \sigma) U^{*}\right] .
$$

Now we shall consider the problem of the mathematical characterization of all possible state changes $\rho \rightarrow \rho_{B}$ caused by some measuring processes of the observable $X$. We define a map $\mathscr{I}$ on the Borel $\sigma$-field $\mathscr{B}(\boldsymbol{R})$ of the real line with values in the transformations on the space $\mathscr{T}\left(\mathscr{H}_{I}\right)$ of all trace class operators on $\mathscr{H}_{I}$ by the relation

$$
\mathscr{I}(B) \rho=\operatorname{Tr}^{I I}\left[U(\rho \otimes \sigma) U^{*}(1 \otimes \tilde{X}(B))\right],
$$

for all $B$ in $\mathscr{B}(\boldsymbol{R})$ and $\rho$ in $\mathscr{T}\left(\mathscr{H}_{I}\right)$. Then $\mathscr{I}$ has the following properties:

(2.11) For each $B$ in $\mathscr{B}(\boldsymbol{R}), \mathscr{I}(B)$ is a completely positive linear map on $\mathscr{T}\left(\mathscr{H}_{I}\right)$.

(2.12) For each countable family $\left\{B_{i}\right\}$ of disjoint Borel sets in $\mathscr{B}(\mathbb{R})$,

$$
\mathscr{I}\left(\cup_{i} B_{i}\right)=\sum_{i} \mathscr{I}\left(B_{i}\right)
$$

where the sum is convergent in the strong operator topology on the space $\mathscr{L}\left(\mathscr{T}\left(\mathscr{H}_{I}\right)\right)$ of all bounded linear transformations on $\mathscr{T}\left(\mathscr{H}_{1}\right)$.

(2.13) For each $\rho$ in $\mathscr{T}\left(\mathscr{H}_{I}\right), \operatorname{Tr}[\mathscr{I}(\boldsymbol{R}) \rho]=\operatorname{Tr}[\rho]$.

(2.14) For each $B$ in $\mathscr{B}(\boldsymbol{R}), \mathscr{I}(B)^{*} 1=X(B)$, where $\mathscr{I}(B)^{*}: \mathscr{L}\left(\mathscr{H}_{I}\right) \rightarrow \mathscr{L}\left(\mathscr{H}_{I}\right)$ is the dual map of $\mathscr{I}(B): \mathscr{T}\left(\mathscr{H}_{I}\right) \rightarrow \mathscr{T}\left(\mathscr{H}_{I}\right)$.

We shall call any map $\mathscr{I}: \mathscr{B}(\boldsymbol{R}) \rightarrow \mathscr{L}\left(\mathscr{T}\left(\mathscr{H}_{I}\right)\right)$ with the above properties (2.11)(2.13) a CP-instrument (cf. Davies-Lewis [7] and Davies [6]). A CPinstrument $\mathscr{I}$ is called $X$-compatible if it further enjoys the property (2.14) (cf. [13; Sect. 4]). We used in [13], the notations $\rho \mathscr{I}(B)$ and $\mathscr{I}(B) a$, for $\rho$ in $\mathscr{T}\left(\mathscr{H}_{I}\right)$ and $a$ in $\mathscr{L}\left(\mathscr{H}_{I}\right)$, instead of $\mathscr{I}(B) \rho$ and $\mathscr{I}(B)^{*} a$ respectively in this paper.

The first answer to the characterization problem is the following.

Theorem 2.1. Let $X$ be an observable in $I$. The relation

$$
\rho_{B}=(1 / \operatorname{Tr}[\rho X(B)]) \mathscr{I}(B) \rho,
$$

for all density operators $\rho$ in $\mathscr{H}_{I}$ and $B$ in $\mathscr{B}(\mathbb{R})$ such that $\operatorname{Tr}[\rho X(B)] \neq 0$, sets up a one-to-one correspondence between all possible state changes $\rho \rightarrow \rho_{B}$ caused by some measuring processes of the observable $X$ in I and all X-compatible $C P$-instruments $\mathscr{I}$. 
Proof. Let $\rho \rightarrow \rho_{B}$ be the state change caused by a measuring process $\left\langle\mathscr{H}_{I I}, \tilde{X}, \sigma, U\right\rangle$ of $X$. Then Eq. (2.10) determines an $X$-compatible $C P$ instrument $\mathscr{I}$ satisfying Eq. (2.15). Conversely, let $\mathscr{I}$ be any $X$-compatible $C P$-instrument. Then by [13; Theorem 5.1, Corollary 5.2], we can construct a measuring process $\left\langle\mathscr{H}_{I I}, \tilde{X}, \sigma, U\right\rangle$ of $X$ satisfying Eq. (2.10) and Eq. (2.15).

Q.E.D.

A completely positive linear map $T: \mathscr{T}\left(\mathscr{H}_{I}\right) \rightarrow \mathscr{T}\left(\mathscr{H}_{I}\right)$ is called an operation if it is trace preserving, i.e.,

$$
\operatorname{Tr}[T \rho]=\operatorname{Tr}[\rho],
$$

for any $\rho$ in $\mathscr{T}\left(\mathscr{H}_{I}\right)$. Denote by $T^{*}$ the dual map $T^{*}: \mathscr{L}\left(\mathscr{H}_{I}\right) \rightarrow \mathscr{L}\left(\mathscr{H}_{I}\right)$ of $T$; then $T^{*}$ is a unit preserving normal completely positive linear map on $\mathscr{L}\left(\mathscr{H}{ }_{I}\right)$. An operation $T$ is called $X$-compatible if the range of $T^{*}$ is contained in $\{X(B)$; $B \in \mathscr{B}(\boldsymbol{R})\}^{\prime}$, or equivalently $T\left[e^{-i t X} \rho e^{i t X}\right]=T[\rho]$ for all $t$ in $\boldsymbol{R}$. The second answer to the characterization problem is simpler than the first as follows.

Theorem 2.2. Let $X$ be an observable in I. The relation

$$
\rho_{B}=T[\rho X(B)] / \operatorname{Tr}[\rho X(B)]
$$

for all density operators $\rho$ on $\mathscr{H}_{I}$ and all $B$ in $\mathscr{B}(\boldsymbol{R})$ such that $\operatorname{Tr}[\rho X(B)] \neq 0$, sets up a one-to-one correspondence between all possible state changes $\rho \rightarrow \rho_{B}$ caused by some measuring processes of an observable $X$ and all $X$-compatible operations $T$.

Proof. The assertion follows from [13; Proposition 4.4, Theorem 2.1]; see also [13; Theorem 5.5]. (In [13], the notation Ta for all $a$ in $\mathscr{L}\left(\mathscr{H}_{I}\right)$ is used instead of $T^{*} a$ in this paper.)

Q.E.D.

As a corollary we can simplify Eq. (2.8) which determines $\rho_{B}$.

Corollary 2.3. For any measuring process $\left\langle\mathscr{H}_{I I}, \tilde{X}, \sigma, U\right\rangle$ of an observable $X$ in $I$, we have

$$
\rho_{B}=(1 / \operatorname{Tr}[\rho X(B)]) \operatorname{Tr}^{I I}\left[U(\rho X(B) \otimes \sigma) U^{*}\right],
$$

for any density operator $\rho$ in $\mathscr{H}_{I}$ and $B$ in $\mathscr{B}(\boldsymbol{R})$ such that $\operatorname{Tr}[\rho X(B)] \neq 0$.

Proof. Let $T$ be an $X$-compatible operation satisfying Eq. (2.17). Then putting $B=\boldsymbol{R}$, we have by Eq. (2.9)

$$
T[\rho]=\rho_{\boldsymbol{R}}=\operatorname{Tr}^{I I}\left[U(\rho \otimes \sigma) U^{*}\right],
$$


for all density operator $\rho$ and hence we have

$$
T[\rho X(B)]=\operatorname{Tr}^{I I}\left[U(\rho X(B) \otimes \sigma) U^{*}\right],
$$

whence Eq. (2.18) follows from Eq. (2.17).

Q.E.D.

\section{§3. A Posteriori States in Quantum Measuring Processes}

In the preceding section, we have determined the state change $\rho \rightarrow \rho_{B}$ caused by a measuring process of an observable $X$. In the conventional theory of quantum measurements for discrete observables, the state change $\rho \rightarrow \rho_{\boldsymbol{B}}$ can be described by the state change $\rho \rightarrow \rho_{x}$ indexed by the result $x$ of the measurement, as follows. Let $X=\sum_{i} x_{i}\left|\xi_{i}><\xi_{i}\right|$ be an observable with simple discrete spectrum $x_{1}, x_{2}, \cdots$. Then the conventional ideal measurement leads the state change $\rho \rightarrow \rho_{x_{i}}$ such that $\rho_{x_{i}}=\left|\breve{\zeta}_{i}\right\rangle\left\langle\zeta_{i}\right|$ for all $i$. In this case, the state change of the form $\rho \rightarrow \rho_{B}$ is obtained by

$$
\rho_{B}=(1 / \operatorname{Prob}(\tilde{X} \in B)) \sum_{x_{i} \in B} \operatorname{Prob}\left(\tilde{X}=x_{i}\right) \rho_{x_{i}},
$$

since $\rho_{x_{1}}=\rho_{\left\{x_{1}\right\}}$ (cf. [13; §9]). In this section, we shall consider the continuous analogue of this type of state changes $\rho \rightarrow \rho_{x}$. Since we allow the observable with continuous spectrum, we cannot hope that Eq. (3.1) is retained; because it may occur that $\operatorname{Prob}(\tilde{X}=x)=0$ for all $x$ in $\mathbb{R}$. Moreover, we can neither hope that $\rho_{x}$ is uniquely determined. But as shown in the following, the problem has much similar analogy in the usual probability theory of continuous random variables. We shall refer to $[4$; Chapter 4$]$ for the background ideas in probability theory.

Our problem is to determine a family $\left\{\rho_{x} ; x \in \mathbb{R}\right\}$ of density operators unique up to almost everywhere with respect to the probability distribution of results of a measurement. Of course, the families $\left\{\rho_{x} ; x \in \mathbb{R}\right\}$ and $\left\{\rho_{B} ; B \in \mathscr{B}(\mathbb{R})\right\}$ must have plausible connection under statistical interpretation of quantum mechanics. Consider the whole ensemble $S$ at the instant after the measurement of an observable $X$. Let $\cdots,\left[x_{-1}, x_{0}\right),\left[x_{0}, x_{1}\right),\left[x_{1}, x_{2}\right), \cdots$ be a partition of $\mathbb{R}$. Then we can divide the ensemble $S$ into the sequence $\cdots, S_{\left[x_{-1}, x_{0}\right)}, S_{\left[x_{0}, x_{1}\right)}, S_{\left[x_{1}, x_{2}\right)}, \cdots$ of subensembles, where $S_{\left[x_{n}, x_{n+1}\right)}$ is the subensemble of $S$ in which the results of the measurement lie in $\left[x_{n}, x_{n+1}\right)$. If the error of this measurement is less than $\varepsilon$ then we can take $x_{n}$ 's so that $x_{n+1}-x_{n}<\varepsilon$. Since we can suppose to get the value of the pointer position $\tilde{X}$ in an arbitrary small error $\varepsilon$, we can divide the ensemble $S$ into $S_{\left[x_{n}, x_{n+1}\right)}$ 's with $x_{n+1}-x_{n}<\varepsilon$ for arbitrary small $\varepsilon$. Thus we can 
expect to make a limit process

$$
\left\{S_{\left[x_{n}, x_{n+1}\right)} ; n=0, \pm 1, \pm 2, \cdots\right\} \longrightarrow\left\{S_{x} ; x \in R\right\}
$$

as $\varepsilon \rightarrow 0$. In this limit process, $S_{x}$ will be a limit of the sequence of the subensembles $S_{\left[x_{n}, y_{n}\right)}$ with $x_{n} \rightarrow x, y_{n} \rightarrow x$ and $x \in\left[x_{n}, y_{n}\right)$. In symbols, we can write

$$
S_{x}=\lim _{\varepsilon \downarrow 0} S_{[x, x+\varepsilon)},
$$

and in this case $S_{x}$ will be the subensemble of $S$ in which the result of the measurement is $x$, i.e., $\tilde{X}=x$. Now we can regard $\rho_{x}$ as the state of the ensemble $S_{x}$ at the instant after the measurement. Then by Eq. (3.2), we have in symbols

$$
\operatorname{Tr}\left[\rho_{x} Y(A)\right]=\lim _{\varepsilon \downarrow 0} \operatorname{Tr}\left[\rho_{[x . x+\varepsilon)} Y(A)\right],
$$

for an arbitrary observable $Y$ in $I$ and $A$ in $\mathscr{B}(\boldsymbol{R})$. By Eq. (2.6) and Eq. (2.7) we have

$$
\begin{aligned}
& \lim _{\varepsilon \downarrow 0} \operatorname{Tr}\left[\rho_{[x, x+\varepsilon)} Y(A)\right] \\
& \quad=\lim _{\varepsilon \downarrow 0} \operatorname{Prob}(Y \in A, \tilde{X} \in[x, x+\varepsilon)) / \operatorname{Prob}(\tilde{X} \in[x, x+\varepsilon)) .
\end{aligned}
$$

Then the right hand side of Eq. (3.4) is the intuitive definition of the conditional probability $\operatorname{Prob}(Y \in A \mid \tilde{X}=x)$ (cf. [4; p. 68, Eq. (4.4)]), i.e.,

$$
\begin{aligned}
& \operatorname{Prob}(Y \in A \mid \tilde{X}=x) \\
& \quad=\lim _{\varepsilon \downarrow 0} \operatorname{Prob}(Y \in A, \tilde{X} \in[x, x+\varepsilon)) / \operatorname{Prob}(\tilde{X} \in[x, x+\varepsilon)) .
\end{aligned}
$$

Thus combining Eqs. (3.3)-(3.5) we obtain the following statistical interpretation of the state $\rho_{x}$ :

$$
\operatorname{Prob}(Y \in A \mid \tilde{X}=x)=\operatorname{Tr}\left[\rho_{x} Y(A)\right],
$$

for any observable $Y$ in $I$ and $A$ in $\mathscr{B}(\boldsymbol{R})$. Unfortunately, the limits in Eqs. (3.3)(3.5) does not exist in general, and hence these equations have only intuitive meanings. In probability theory the precise definition of the conditional probability $\operatorname{Prob}(Y \in A \mid \tilde{X}=x)$ is a Borel function $x \rightarrow \operatorname{Prob}(Y \in A \mid \tilde{X}=x)$ such that

$$
\int_{B} \operatorname{Prob}(Y \in A \mid \tilde{X}=x) \operatorname{Prob}(\tilde{X} \in d x)=\operatorname{Prob}(Y \in A, \tilde{X} \in B),
$$

for any Borel set $B$ in $R$. Eq. (3.7) determines a function $x \rightarrow \operatorname{Prob}(Y \in A \mid \tilde{X}=x)$ unique up to almost everywhere with respect to $\operatorname{Prob}(\tilde{X} \in d x)$. Thus we can determine only a function $x \rightarrow \rho_{x}$ unique up to almost everywhere with respect to 
$\operatorname{Prob}(\tilde{X} \in d x)$.

Our precise definition of the required family $\left\{\rho_{x} ; x \in \mathbb{R}\right\}$ is as follows:

(A1) For any $x$ in $\boldsymbol{R}, \rho_{x}$ is a density operator on $\mathscr{H}_{I}$.

(A2) The function $x \rightarrow \rho_{x}$ is strongly Borel measurable.

(A3) For any observable $Y$ in $I$ and any Borel sets $A$ and $B$.

$$
\int_{B} \operatorname{Tr}\left[\rho_{x} Y(A)\right] \operatorname{Prob}(\tilde{X} \in d x)=\operatorname{Prob}(Y \in A, \tilde{X} \in B) .
$$

We call any family $\left\{\rho_{x} ; x \in \mathbb{R}\right\}$ of density operators on $\mathscr{H}_{I}$ satisfying Conditions (A1)-(A3) a family of a posteriori states for an a priori state $\rho$ with respect to a measuring process $\left\langle\mathscr{H}_{I I}, \tilde{X}, \sigma, U\right\rangle$ of an observable $X$ in $I$.

Let $\mathscr{I}$ be the CP-instrument corresponding to the measuring process $\left\langle\mathscr{H}_{I I}, \tilde{X}, \sigma, U\right\rangle$. Then Condition (A3) is equivalent to

(A3') For any observable $Y$ in $I$ and any Borel sets $A$ and $B$,

$$
\int_{B} \operatorname{Tr}\left[\rho_{x} Y(A)\right] \operatorname{Tr}[\mathscr{I}(d x) \rho]=\operatorname{Tr}[(\mathscr{I}(B) \rho) Y(A)]
$$

Thus the family of a posteriori states is completely determined by the CPinstrument corresponding to the measuring process.

If there is a family of a posteriori states then by Eq. (3.6), the conditional expectation $\operatorname{Ex}(Y \mid \tilde{X}=x)$ of $Y$ with respect to $X$ is obtained by the following relations:

$$
\begin{aligned}
\operatorname{Ex}(Y \mid \tilde{X}=x) & =\int_{\mathbb{R}} y \operatorname{Prob}(Y \in d y \mid \tilde{X}=x) \\
& =\int_{\mathbb{R}} y \operatorname{Tr}\left[\rho_{x} Y(d y)\right] \\
& =\operatorname{Tr}\left[\rho_{x} Y\right],
\end{aligned}
$$

(cf. [4; p. 79, Proposition 4.35]).

Now we shall prove the existence of a family of a posteriori states for any measuring process.

Theorem 3.1. For any observable $X$ in I, any measuring process $\left\langle\mathscr{H}_{I I}\right.$, $\tilde{X}, \sigma, U>$ of $X$ and any density operator $\rho$ on $\mathscr{H}_{1}$, there is a family $\left\{\rho_{x}\right.$; $x \in \mathbb{R}\}$ of a posteriori states for the a priori state $\rho$.

Proof. Let $\mu$ be a probability measure on $\mathbb{R}$ such that $\mu(d x)=\operatorname{Tr}[\rho X(d x)]$. By Eq. (2.3), $\mu(B)=0$ if and only if $\operatorname{Tr}\left[U(\rho \otimes \sigma) U^{*}(1 \otimes \tilde{X}(B))\right]=0$ for all $B$ in $\mathscr{B}(\boldsymbol{R})$. Thus by the spectral multiplicity theory, there is a projection $E$ in 
$\tilde{X}(\mathscr{B}(\boldsymbol{R}))^{\prime \prime}$ such that $E \tilde{X}(\mathscr{B}(\boldsymbol{R}))^{\prime \prime} \simeq L^{\infty}(\boldsymbol{R}, \mu)$, and that $\operatorname{Tr}\left[U(\rho \otimes \sigma) U^{*}\left(1 \otimes E^{\perp}\right)\right]=0$. Thus we can extend the map $a \otimes f \rightarrow a \otimes E \tilde{X}(f)$ for $a$ in $\mathscr{L}\left(\mathscr{H}_{I}\right)$ and $f$ in $L^{\infty}(\boldsymbol{R}, \mu)$ to a normal *-representation of $\mathscr{L}\left(\mathscr{H}_{I}\right) \otimes L^{\infty}(\boldsymbol{R}, \mu)$, where $E \tilde{X}(f)=\int_{R} f(x)$. $E \tilde{X}(d x)$. It follows that the relation

$$
\rho^{\prime \prime}(a \otimes f)=\operatorname{Tr}\left[U(\rho \otimes \sigma) U^{*}(a \otimes E \tilde{X}(f))\right],
$$

for all $a$ in $\mathscr{L}\left(\mathscr{H}_{I}\right)$ and $f$ in $L^{\infty}(\boldsymbol{R}, \mu)$, defines a normal state on $\mathscr{L}\left(\mathscr{H}_{I}\right) \otimes L^{\infty}(\boldsymbol{R}, \mu)$. Then by [16; Theorem IV.7.17], there is a family $\left\{\rho_{x} ; x \in \mathbb{R}\right\}$ in $L^{1}\left(\boldsymbol{R}, \mu, \mathscr{T}\left(\mathscr{H}_{I}\right)\right)$ such that

$$
\rho^{\prime \prime}\left(a \otimes \chi_{B}\right)=\int_{B} \operatorname{Tr}\left[\rho_{x} a\right] \mu(d x),
$$

for all $a$ in $\mathscr{L}\left(\mathscr{H}_{\mathrm{I}}\right)$ and $B$ in $\mathscr{B}(\boldsymbol{R})$. Since $\rho^{\prime \prime}\left(a \otimes \chi_{B}\right)=\operatorname{Tr}\left[U(\rho \otimes \sigma) U^{*}(a \otimes \tilde{X}(B))\right]$ $=\operatorname{Tr}[\mathscr{F}(B) \rho]$ for all $B$ in $\mathscr{B}(\boldsymbol{R})$, the assertion follows immediately. Q.E.D.

In the next section, we shall consider the concept of a posteriori states in operational quantum probability theory and generalise the above theorem to not necessarily completely positive instruments. This generalisation enables us to resolve the Davies-Lewis conjecture completely.

\section{§4. A Posteriori States in Quantum Probability Theory}

In this section we shall study the concept of a posteriori states within the framework of operational quantum probability theory due to Davies-Lewis [7].

Our setting for operational quantum probability theory consists of a von Neumann algebra $\mathscr{M}$ and a Borel space $\left(\Lambda, \mathscr{B}(\Lambda)\right.$ ). Denote by $\mathscr{M}_{*}$ the predual of $\mathscr{M}$, and by $\mathscr{L}^{+}\left(\mathscr{M}_{*}\right)$ the space of all positive linear transformations on $\mathscr{M}_{*}$. Denote by $\langle\cdot, \cdot\rangle$ the duality pairing between $\mathscr{M}_{*}$ (or $\mathscr{M}^{*}$ ) and $\mathscr{M}$. A map $\mathscr{I}: \mathscr{B}(\Lambda) \rightarrow \mathscr{L}^{+}\left(\mathscr{M}_{*}\right)$ is called an instrument for $(\mathscr{M}, \Lambda)$, if it satisfies the following conditions (I1)-(I2):

(I1) For each $\rho$ in $\mathscr{M}_{*},\langle\mathscr{I}(\Lambda) \rho, 1\rangle=\langle\rho, 1\rangle$.

(I2) For each disjoint sequence $\left\{B_{i}\right\}$ in $\mathscr{B}(\Lambda)$,

$$
\mathscr{I}\left(\cup_{i} B_{i}\right)=\sum_{i} \mathscr{I}\left(B_{i}\right),
$$

where the sum is convergent in the strong operator topology of $\mathscr{L}^{+}\left(\mathscr{M}_{*}\right)$.

An instrument $\mathscr{I}$ is called a $C P$-instrument if it further enjoys the following condition (I3): 
(I3) For each $B$ in $\mathscr{B}(\Lambda), \mathscr{I}(B)$ is completely positive.

Let $\mathscr{I}$ be an instrument. Now we fix a normal state $\rho$ on $\mathscr{M}$. Then we can associate a probability distribution $\mu$ on $(\Lambda, \mathscr{B}(\Lambda))$ such that

$$
\mu(d x)=\langle\mathscr{I}(d x) \rho, 1\rangle .
$$

We call this $\mu$ the probability distribution associated with $(\mathscr{I}, \rho)$.

Definition 4.1. A family $\left\{\rho_{x} ; x \in \Lambda\right\}$ of normal states on $\mathscr{M}$ is called a family of a posteriori states with respect to $(\mathscr{I}, \rho)$ if it has the following properties (F1)-(F2):

(F1) For any $a$ in $\mathscr{\ell}$, the function $x \rightarrow\left\langle\rho_{x}, a\right\rangle$ is $\mu$-measurable.

(F2) For any $a$ in $\mathscr{M}$ and $B$ in $\mathscr{B}(\Lambda)$,

$$
\int_{B}\left\langle\rho_{x}, a\right\rangle \mu(d x)=\langle\mathscr{I}(B) \rho, a\rangle \text {. }
$$

Moreover, it is called proper if it satisfies the following:

(F3) For any $a$ in $\mathscr{M}$ with $a \geq 0$, if $\langle\mathscr{I}(\Lambda) \rho, a\rangle=0$ then $\left\langle\rho_{x}, a\right\rangle=0$ for all $x$ in $\Lambda$.

A family $\left\{\rho_{x} ; x \in \Lambda\right\}$ of a posteriori states is called $\mathscr{B}(\Lambda)$-measurable if the function $x \rightarrow \rho_{x}$ is $\mathscr{B}(\Lambda)$-measurable in the sense that there is a sequence $F_{n}$ of $\mathscr{B}(\Lambda)$-measurable $\mathscr{M}_{*}$-valued simple functions such that $\lim _{n}\left\|\rho_{x}-F_{n}(x)\right\|=0$ for every $x$ in $\Lambda$.

Let $\left\{\rho_{x} ; x \in \Lambda\right\}$ and $\left\{\rho_{x}^{\prime} ; x \in \Lambda\right\}$ be two families of a posteriori states with respect to $(\mathscr{I}, \rho)$. By Condition (F2), they are equivalent in the sense that $\left\langle\rho_{x}, a\right\rangle=\left\langle\rho_{x}^{\prime}, a\right\rangle, \mu$-a.e. for all $a$ in $\mathscr{M}$. If they are both $\mathscr{B}(\Lambda)$-measurable then $\rho_{x}=\rho_{x}^{\prime} \mu$-a.e., since their ranges are separable.

Denote by $M^{\infty}(\mu)$ the space of all bounded complex-valued $\mu$-measurable functions on $\Lambda$. A lifting $\Phi$ of $M^{\infty}(\mu)$ is a $*$-isomorphism from $M^{\infty}(\mu)$ into $M^{\infty}(\mu)$ such that $\Phi^{-1}(0)=\left\{f \in M^{\infty}(\mu) ; f=0 \mu\right.$-a.e. $\}$ and that $\Phi(f)=f \mu$-a.e. for all $f \in M^{\infty}(\mu)$. For the existence of the lifting, we shall refer to [18]. For Banach spaces $A$ and $B$ with $A^{*}=B$ or $A=B^{*}$, denote by $M^{\infty}(\mu, A,[B])$ the vector space of all functions $f: \Lambda \rightarrow A$ having the following properties:

i) $f(\Lambda) \subseteq A$ is relatively $\sigma(A, B)$-compact;

ii) $\langle f(\cdot), \varphi\rangle \in M^{\infty}(\mu)$ for every $\varphi$ in $B$.

For the case $A=B^{*}$, Condition i) follows from Condition ii). If $A$ is separable, 
every function in $M^{\infty}(\mu, A,[B])$ is strongly measurable. We shall also denote by $\Phi$ the linear lifting of $M^{\infty}(\mu, A,[B])$ associated with the lifting $\Phi$ of $M^{\infty}(\mu)$ (cf. [18; pp. 75-76]).

Let $\rho$ be a normal state on $\mathscr{M}$ and $\mathscr{I}$ be an instrument for $(\mathscr{M}, \Lambda)$. For any $a$ in $\mathscr{M}$, and $B$ in $\mathscr{B}(\Lambda)$, we have $|\langle\mathscr{I}(B) \rho, a\rangle| \leq\|a\| \mu(B)$. Thus the function $B \rightarrow\langle\mathscr{I}(B) \rho, a\rangle$ is a finite signed measure on $(\Lambda, \mathscr{B}(\Lambda)$ ) absolutely continuous with respect to $\mu$. Let $f \in L^{1}(\Lambda, \mu)$. Since the $\mathscr{M}_{*}$-valued measure $\mathscr{I}(d x) \rho$ is countably additive in norm, the relation

$$
\langle\mathscr{E}(f), a\rangle=\int_{\Lambda} f(x)\langle\mathscr{I}(d x) \rho, a\rangle
$$

defines a positive linear operator $\mathscr{E}: L^{1}(\Lambda, \mu) \rightarrow \mathscr{M}_{*}$. We have that

$$
\|\mathscr{E}(f)\|=\langle\mathscr{E}(f), 1\rangle=\int_{\Lambda} f(x) \mu(d x)=\|f\|,
$$

for all $f \geq 0$ in $L^{1}(\Lambda, \mu)$, i.e., $\mathscr{E}^{*}(1)=1$.

In the following, we shall give some conditions for the existence of a family of a posteriori states.

Theorem 4.2. Let $\mathscr{I}$ be an instrument for $(\mathscr{M}, \Lambda)$ and $\rho$ a normal state on $\mathscr{M}$. If, for any decreasing sequence $\left\{p_{n}\right\}$ of projections in $\mathscr{M}$ with $\inf _{n} p_{n}=0$, we have

$$
\lim _{n} \int_{\Lambda} f(x)\left\langle\mathscr{I}(d x) \rho, p_{n}\right\rangle=0,
$$

uniformly for all $f$ in $L^{1}(\Lambda, \mu)$ with $\|f\| \leq 1$, then there is a $\mathscr{B}(\Lambda)$-measurable proper family $\left\{\rho_{x} ; x \in \Lambda\right\}$ of a posteriori states with respect to $(\mathscr{I}, \rho)$.

Proof. By assumption and [16; Theorem III.5.4], $\mathscr{E}$ is a weakly compact operator. Thus by the Dunford-Pettis-Phillips theorem [18; p. 89, Corollary 2], we have a family $\left\{\rho_{x} ; x \in \Lambda\right\}$ in $M^{\infty}\left(\mu, \mathscr{M}_{*},[\mathscr{M}]\right)$ such that $\Phi\left(\rho_{x}(a)\right)=\rho_{x}(a)$ and that

$$
\langle\mathscr{E}(f), a\rangle=\int_{\Lambda} f(x) \rho_{x}(a) \mu(d x),
$$

for all $a$ in $\mathscr{M}$. Then it is straightforward to check that $\left\{\rho_{x} ; x \in \Lambda\right\}$ is a proper family of a posteriori states. By [18; Theorem 3, p. 92], it is strongly measurable and hence by redefining $\rho_{x}$ as $\rho_{x}=\mathscr{I}(\Lambda) \rho$ on a $\mu$-null set, we have a $\mathscr{B}(\Lambda)$-measurable family of a posteriori states.

Q.E.D.

Theorem 4.3. Let $\mathscr{M}$ be the enveloping von Neumann algebra of some 
$C^{*}$-algebra $\mathscr{A}$. If the sequential weak closure $\sigma(\mathscr{A})$ of $\mathscr{A}$ in $\mathscr{M}$ coincides with $\mathscr{M}$, then for any instrument $\mathscr{I}$ for $(\mathscr{M}, \Lambda)$ and any normal state $\rho$ on $\mathscr{M}$ there is a proper family $\left\{\rho_{x} ; x \in \Lambda\right\}$ of a posteriori states with respect to $(\mathscr{I}, \rho)$.

Proof. Since $\mathscr{E} \in \mathscr{L}\left(L^{1}(\Lambda, \mu), \mathscr{A}^{*}\right)$, by the Dunford-Pettis theorem $[18$, p. 89 , Corollary 1$]$, we have a family $\left\{\rho_{x} ; x \in \Lambda\right\}$ in $M^{\infty}\left(\mu, \mathscr{A}^{*},[\mathscr{A}]\right)$ such that $\Phi\left(\rho_{x}(a)\right)=\rho_{x}(a)$ and that

$$
\langle\mathscr{E}(f), a\rangle=\int_{\Lambda} f(x) \rho_{x}(a) \mu(d x)
$$

for all $a$ in $\mathscr{A}$. By the relation $\Phi\left(\rho_{x}(a)\right)=\rho_{x}(a)$, and by the relation $\mathscr{A}^{*}=\mathscr{M}_{*}$, $\rho_{x}$ is a normal positive linear functional on $\mathscr{M}$ for all $x$ in $\Lambda$. By the bounded convergence theorem, the set of all $a$ in $\mathscr{M}$ for which Eq. (4.1) holds contains the weak sequential closure of $\mathscr{A}$, and hence we have

$$
\int_{\Lambda} \rho_{x}(1) \mu(d x)=\langle\mathscr{E}(1), 1\rangle=\langle\mathscr{I}(\Lambda) \rho, 1\rangle=1 .
$$

Thus there is a $\mu$-null set $N$ such that $\rho_{x}(1)=1$ on $A-N$. Redefine $\rho_{x}$ for $x$ in $N$ as $\rho_{x}=\mathscr{I}(\Lambda) \rho$. Then it is obvious that $\left\{\rho_{x} ; x \in \Lambda\right\}$ is a required family of a posteriori states.

Q.E.D.

Remark. The assumption $\sigma(\mathscr{A})=\mathscr{A}^{* *}$ is statisfied if

(1) $\mathscr{A}^{*}$ is separable, or

(2) $\mathscr{A}$ is separable and the center of $\mathscr{A}^{* *}$ is countably decomposable (cf. [21]).

If $\mathscr{A}^{*}$ is separable then the family $\left\{\rho_{x} ; x \in \Lambda\right\}$ is strongly measurable and hence we can obtain a $\mathscr{B}(\Lambda)$-measurable proper family of a posteriori states. The case $\mathscr{M}=\mathscr{L}(\mathscr{H})$ for separable $\mathscr{H}$ is included in this case, so that we have reproved Theorem 3.1.

Theorem 4.4. Let , ll be the enveloping von Neumann algebra of some $C^{*}$-algebra $\mathscr{A}$. If $\mathscr{A}$ is an ideal of $\mathscr{C l}$, then there is a proper family $\left\{\rho_{x} ; x \in \Lambda\right\}$ of a posteriori states with respect to $(\mathscr{I}, \rho)$.

Proof. Since $\mathscr{E} \in \mathscr{L}\left(L^{1}(\Lambda, \mu), \mathscr{M}^{*}\right)$, by the Dunford-Pettis theorem [18; p. 89, Corollary 1$]$, we have a family $\left\{\rho_{x} ; x \in \Lambda\right\}$ in $M^{\infty}\left(\mu, \mathscr{M}^{*},\left[\mathscr{M}^{\prime}\right]\right)$ such that $\Phi\left(\rho_{x}(a)\right)=\rho_{x}(a)$ and that

$$
\langle\mathscr{E}(f), a\rangle=\int_{\Lambda} f(x) \rho_{x}(a) \mu(d x),
$$

for all $a$ in $\mathscr{M}$. Let $\mathscr{M}^{\frac{1}{*}}$ be the singular part of $\mathscr{M}^{*}$. Since $\mathscr{A}$ is an ideal of 
$\mathscr{M}$, we have $\mathscr{M}_{*}^{+}=\mathscr{A}^{\circ}$ in $\mathscr{M}^{*}$, where $\mathscr{A}^{\circ}$ is the polar of $\mathscr{A}$ (cf. [16; Proposition III.2.17]). Let $\left\{u_{\alpha}\right\}$ be an increasing approximate identity in $\mathscr{A}$. Then we have

$$
\rho_{x}^{n}(1)=\rho_{x}^{n}\left(\sup _{\alpha} u_{\alpha}\right)=\sup _{\alpha} \rho_{x}^{n}\left(u_{\alpha}\right)=\sup _{\alpha} \rho_{x}\left(u_{\alpha}\right),
$$

where $\rho_{x}^{n}$ stands for the normal part of $\rho_{x}$ and the last equality follows from $\mathscr{M}_{*}^{\perp}=\mathscr{A}^{\circ}$. Since $\Phi\left(\rho_{x}\left(u_{\alpha}\right)\right)=\rho_{x}\left(u_{\alpha}\right)$, the function $x \rightarrow \sup _{\alpha} \rho_{x}\left(u_{\alpha}\right)$ is $\mu$-measurable and

$$
\int_{\Lambda} \sup _{\alpha} \rho_{x}\left(u_{\alpha}\right) \mu(d x)=\sup _{\alpha} \int_{\Lambda} \rho_{x}\left(u_{\alpha}\right) \mu(d x),
$$

by $[18 ;$ p. 40, Theorem 3$]$. Thus we have

$$
\begin{aligned}
\int_{\Lambda}\left\|\rho_{x}^{n}\right\| \mu(d x) & =\int_{\Lambda} \rho_{x}^{n}(1) \mu(d x) \\
& =\sup _{\alpha} \int_{\Lambda} \rho_{x}\left(u_{\alpha}\right) \mu(d x) \\
& =\sup _{\alpha}\left\langle\mathscr{I}(\Lambda) \rho, u_{\alpha}\right\rangle=\langle\mathscr{I}(\Lambda) \rho, 1\rangle=1 .
\end{aligned}
$$

It follows that $\rho_{x}^{n}=\rho_{x} \mu$-a.e., so that there is a $\mu$-null set $N$ such that $\rho_{x}$ is a normal state for all $x$ in $\Lambda-N$. By redefining $\rho_{x}$ as $\rho_{x}=\mathscr{I}(\Lambda) \rho$ on $N$, the conclusion follows immediately.

Q.E.D.

Remark. The condition that $\mathscr{A}$ is an ideal in $\mathscr{A}^{* *}$ is equivalent to that $\mathscr{A}$ is isomorphic to $C(\infty)$-direct sum of a family of the $C^{*}$-algebras of compact operators on Hilbert spaces (cf. $[16 ;$ p. 157]). Thus the case $\mathscr{M}=\mathscr{L}(\mathscr{H})$ is included for any Hilbert space $\mathscr{H}$.

Theorem 4.5. Let $\mathscr{M}=\mathscr{L}(\mathscr{H})$ for some Hilbert space $\mathscr{H}$, and $\mathscr{I}$ be a CPinstrument for $(\mathscr{M}, \Lambda)$. Then for any normal state $\rho$ on $\mathscr{M}$, there is a $\mathscr{B}(\Lambda)$ measurable family $\left\{\rho_{x} ; x \in \Lambda\right\}$ of a posteriori states with respect to $(\mathscr{I}, \rho)$.

Proof. By [13; Theorem 5.1] the proof of Theorem 3.1 works. Q.E.D.

In spite of the existence for the case $\mathscr{H}=\mathscr{L}(\mathscr{H})$, the following example suggests that some classes of instruments do not have its family of a posteriori states. Let $P$ be the Lebesgue measure on $[0,1]$, and let $\mathscr{I}$ be an instrument for $\left\langle L^{\infty}([0,1], P),[0,1]\right\rangle$ such that $\mathscr{I}(B) f=\chi_{B} f$ for all Borel set $B$ in $[0,1]$ and $f$ in $L^{1}([0,1], P)$. Then $\mu=P$ for $\rho=1$ in $L^{1}([0,1])$. If $\left\{\rho_{\lambda} \in L^{1}([0,1], P)\right.$; $x \in[0,1]\}$ is a family of a posteriori states, then by Condition $(\mathrm{F} 2)$ we have for all $g$ in $C([0,1])$

$$
\int_{0}^{1} g(y) \rho_{x}(y) P(d y)=g(x) \quad \text { a.e. }
$$


Since $C([0,1])$ is separable, we can choose a common null set outside of which the above equality holds for all $g$ in $C([0,1])$. However, this contradicts the fact that $\rho_{x} \in L^{1}([0,1])$. Thus the above instrument do not have a family of a posteriori states with respect to $\rho=1$.

The above consideration will be generalized in the next section, and we prove that non-discrete weakly repeatable instruments have no families of a posteriori states.

\section{§5. Repeatability}

In [7], Davies and Lewis conjectured the non-existence of repeatable instruments for continuous observables in the standard formulation of quantum mechanics. We have proved this conjecture among CP-instruments in [13]. In this section, we shall show the close connection between the existence of a family of a posteriori states and the non-existence of non-discrete weakly repeatable instruments and as a consequence we shall resolve Davies and Lewis's conjecture completely.

Let $\mathscr{H}$ be a von Neumann algebra and $(\Lambda, \mathscr{B}(\Lambda))$ be a standard Borel space. $\Lambda \mathrm{n}$ instrument $\mathscr{I}$ for $(\mathscr{M}, \Lambda)$ is called discrete if there is a countable subset $\Lambda_{0}$ such that $\mathscr{I}\left(\Lambda-\Lambda_{0}\right)^{*}=0$. An instrument $\mathscr{I}$ is called weakly repeatable if $\mathscr{I}(B)^{*} \mathscr{I}(C)^{*} 1=\mathscr{I}(B \cap C)^{*} 1$ for all $B, C$ in $\mathscr{B}(\Lambda)$.

Theorem 5.1. Let $\mathscr{M}$ be a von Neumann algebra and $(\Lambda, \mathscr{B}(\Lambda)$ ) be a standard Borel space. Let $\mathscr{I}$ be a weakly repeatable instrument for $(\mathscr{M}, \Lambda)$. If, for a faithful normal state $\rho$ on $\mathscr{M}$, there is a family $\left\{\rho_{x} ; x \in \Lambda\right\}$ of a posteriori states with respect to $(\mathscr{I}, \rho)$, then $\mathscr{I}$ is discrete.

Proof. Let $\rho$ be a faithful normal state on $\mathscr{C l}$ and $\left\{\rho_{x} ; x \in \Lambda\right\}$ a family of a posteriori states with respect to $(\mathscr{I}, \rho)$, where $\mathscr{I}$ is weakly repeatable. Let $X(d x)=\mathscr{I}(d x)^{*} 1$ and $\mu(d x)=\langle\rho, X(d x)\rangle$ and let $\left\{B_{n} ; n=1,2, \cdots\right\}$ be a countable subfield of $\mathscr{B}(\Lambda)$ which generates $\mathscr{B}(\Lambda)$. Denote by $\delta_{x}$ the point mass of $x$ in $\Lambda$. By the weak repeatability of $\mathscr{I}$, we have for all $B$ in $\mathscr{B}(\Lambda)$ and all $n$,

$$
\begin{gathered}
\int_{B} \delta_{x}\left(B_{n}\right) \mu(d x)=\mu\left(B \cap B_{n}\right)=\left\langle\rho, X\left(B \cap B_{n}\right)\right\rangle \\
=\left\langle\mathscr{I}(B) \rho, X\left(B_{n}\right)\right\rangle=\int_{B}\left\langle\rho_{x}, X\left(B_{n}\right)\right\rangle \mu(d x) .
\end{gathered}
$$

It follows that for any $n$, there is a $\mu$-null set $N_{n}$ such that $\left\langle\rho_{x}, X\left(B_{n}\right)\right\rangle=\delta_{x}\left(B_{n}\right)$ 
for any $x$ in $\Lambda-N_{n}$. Let $\Lambda_{0}=\Lambda-\cup_{n=1}^{\infty} N_{n}$. Then $\mathscr{I}\left(\Lambda-\Lambda_{0}\right)=0$, since $\rho$ is faithful. Let $x \in \Lambda_{0}$. Since $\rho_{x}$ is a normal state, $B \rightarrow\left\langle\rho_{x}, X(B)\right\rangle$ is a probability measure and hence we have $\left\langle\rho_{x}, X(B)\right\rangle=\delta_{x}(B)$ for all $B$ in $\mathscr{B}(\Lambda)$. It follows that $\left\langle\rho_{x}, X(\{x\})\right\rangle=1$ so that $X(\{x\}) \neq 0$ for all $x$ in $\Lambda_{0}$. Since $\rho$ is faithful, we have $\langle\rho, X(\{x\})\rangle \neq 0$ for all $x$ in $\Lambda_{0}$. Thus $\Lambda_{0}$ is a countable set.

Q.E.D.

Remark. In the above proof we have only to assume the faithfulness of $\rho$ on $\left\{\mathscr{I}^{*}(B) 1 ; B \in \mathscr{B}(\Lambda)\right\}$.

Theorem 5.2. Let $\mathscr{H}$ be a separable Hilbert space and $(\Lambda, \mathscr{B}(\Lambda))$ a standard Borel space. Every weakly repeatable instrument $\mathscr{I}$ for $(\mathscr{L}(\mathscr{H}), \Lambda)$ is discrete.

Proof. Since $\mathscr{H}$ is separable, there is a faithful normal state $\rho$ on $\mathscr{L}(\mathscr{H})$. By Theorem 4.3, there is a family $\left\{\rho_{x} ; x \in \Lambda\right\}$ of a posteriori states with respect to $(\mathscr{I}, \rho)$. Thus by Theorem 5.1 , any weakly repeatable instrument $\mathscr{I}$ is discrete.

Q.E.D.

\section{Acknowledgements}

The author wishes to thank Professor H. Umegaki for useful comments and encouragement. He is also grateful to Professor A. S. Holevo and Professor E. B. Davies for stimulating discussions. The author wishes to thank the referee for his helpful comments and suggestions for improving the first version.

\section{References}

[1] Araki, H., Yanase, M. M., Measurement of quantum mechanical operators. Phy's. Rev. 120 (1960), 622-626.

[2] Arveson, W. B., Analyticity in operator algebras. Amer. J. Math. 89 (1967), 578-642.

[3] Berberian, S. K., Notes on spectral theory. Princeton, Van Nostrand, 1966.

[4] Breiman, L., Prohability. London, Addison-Wesley, 1968.

[5] Cycon, H., Hellwig, K. -E., Conditional expectations in generalized probability theory. I. Math. Phys. 18 (1977), 1154-1161.

[6] Davies, E. B., Quantum theory of open systems. London, Academic Press, 1976.

[7] Davies, E. B., Lewis, J. T., An operational approach to quantum probability. Commun. Math. Phys. 17 (1970), 239-260.

[8] Holevo, A.S., Statistical decision theory for quantum systems. J. Multivar. Anal. 3 (1973), 337-394. 
[9] Holevo, A. S., Probabilistic and statistical aspects of quantum theory. Amsterdam, North-Holland, 1982.

[10] Kraus, K., General state changes in quantum theory. Ann. Phys. 64 (1971), 311-335.

[11] Nakamura, M., Umegaki, H., On von Neumann's theory of measurements in quantum statistics. Math. Japonica 7 (1962), 151-157.

[12] von Neumann, J., Mathematical foundations of quantum mechanics. Princeton, Princeton Univ. Press, 1955.

[13] Ozawa, M., Quantum measuring processes of continuous observables. J. Math. Phys. 25 (1984), 79-87.

[14] Schatten, R., Norm ideals of completely continuous operators. Berlin, Springer, 1960.

[15] Schwartz, L., Lectures on disintegration of measures. Bombay, Tata Institute of Fundamental Research, 1975.

[16] Takesaki, M., Theory of operator algebras I. New York, Springer, 1979.

[17] Tomiyama, J., On the projection of norm one in $W^{*}$-algebra. Proc. Japan Acad. 33 (1957), 608-612.

[18] Tulcea, I. T., Tulcea, I. C., Topics in the theory of lifting. New York, Springer, 1969.

[19] Umegaki, H., Conditional expectation in an operator algebra I, II, III, IV, Tohoku Math. J. 6 (1954), 177-181, 8 (1956), 86-100, Kodai Math. Sem. Rep. 11 (1959), 51-74, 14 (1962), 59-85.

[20] Wigner, E. P., Die Messung Quantenmechanischer Operatoren. Z. Phys. 133 (1952), 101-108.

[21] Davies, E. B., On the Borel structure of $C^{*}$-algebras, Commun. Math. Phys. 8 (1959), 147-168, with Appendix by R. V. Kadison. 
